\title{
DETERMINATION OF OPERATING POLICIES FOR A BARGE TRANSPORTATION SYSTEM THROUGH SIMULATION AND OPTIMIZATION MODELING
}

\author{
Nicholas P. Anderson \\ Mesoscale Diagnostics \\ Gaithersburg, MD 20877
}

Gerald W. Evans

Dept. of Industrial Engineering

University of Louisville

Louisville, KY 40292

\begin{abstract}
This paper presents a simulation model of a barge transportation system for petroleum delivery within an inland waterway. The simulation is employed as an evaluation model within a decision support system which also includes a criterion model, represented as a decision maker's utility function, and an optimization procedure which employs scatter search. Variance reduction techniques are also employed in order to improve the accuracy of the estimates of the performance measures associated with the system. The main purpose of the system is to determine values for important inventory policy variables.
\end{abstract}

\section{INTRODUCTION}

Simulation is a useful methodology for the evaluation of policies related to complex dynamic, stochastic systems. The use of simulation has its disadvantages however. For example, development of a simulation model is often time consuming and expensive. Additional difficulties have to do with the facts that:

1. Evaluation of a policy must often be based on multiple, conflicting performance measures.

2. Typically one can only attain estimates of these performance measure values.

3. The number of feasible policies for evaluation are often too many for complete enumeration.

Related to the second difficulty above, Law and Kelton (2000) note that "each run of a stochastic simulation produces only estimates of a model's true characteristics".

The third difficulty noted above means that one must often employ a sophisticated optimization procedure in order to find a near optimal control policy. Given that one does not have a closed-form representation of the relationship between the control policy variables and the performance measures (one of the reasons for employing simulation in the first place), standard optimization tech- niques associated with solving linear programs or integer programs are not appropriate for these situations; one must employ a heuristic optimization technique.

This paper describes a simulation-based decision support system for determining operating policies for a barge transportation system on an inland river system. In addition to a simulation model, the system relies on the input of a decision maker's utility function over conflicting performance measures, the use of standard variance reduction techniques, and an optimization procedure based on the heuristic optimization procedure, scatter search (Glover and Laguna 2000).

The next section of this paper gives a brief description of the system under study and the associated simulation model. The third section of the paper gives an overview of the decision support system, including a discussion of the utility function, the optimization methodology, and the variance reduction techniques employed. The fourth section of the paper provides an illustrative example of the use of the system. Finally, the fifth section of the paper provides a summary and conclusions.

\section{DESCRIPTION OF THE TRANSPORTATION SYSTEM AND MODEL}

The application was developed to aid a decision maker in a complex, stochastic environment. The Arena software package (Kelton, Sadowski, and Sturrock 2004) was chosen as a base for the DSS. The flexibility of Arena is demonstrated in this research as the DSS includes Arena modules, SIMAN blocks, imbedded Visual Basic for Applications, and is manipulated by external Visual Basic code.

The objective of this study was to determine the best configuration of dedicated tows to deliver volumes of six liquid fuel products to six distinct locations on an inland river system. To accomplish this goal, a determination needed to be made of the number of dedicated boats required to meet the demand for fuel. The fuels are delivered to six locations on the river system. All trips begin 


\section{Anderson and Evans}

and end at a supply location, Node 6 . Nodes 1 through 5 are upriver of Node 6, while Node 7 is downriver. Based on capacity requirements and federal regulations, the configuration of a tow is as follows: a tow is made up of four barges; a barge is made up of ten tanks (two each of five different sizes). A barge can be loaded with either all diesel or all non-diesel fuel, i.e., diesel and non-diesel cannot be mixed within a barge. However, diesel and non-diesel can be mixed within a tow.

The system operated according to a reorder point, order - up-to quantity. That is, the main control variables associated with the inventory policy were the reorder point and the maximum capacity for each storage tank (i.e., demand point).

Initial modeling assumptions included:

1. The supply location, Node 6 , never runs out of fuel.

2. Barges are loaded at a rate of $5000 \mathrm{bbls} / \mathrm{hr}$, one barge at a time.

3. Barges are unloaded at a rate of $2800 \mathrm{bbls} / \mathrm{hr}$, one barge at a time.

The Arena simulation model works as follows: Each fuel level at each location is represented by a variable. Each day an entity is created for each fuel at each location. This entity decrements the level of the variable representing the level of that fuel type for that particular location. If the level of the on-hand inventory is below a set reorder point, a check is made to determine if there is a stockout situation. If there is a stockout, a counter is incremented and a mechanism is invoked to record the amount of time associated with the stockout. The entity can now be thought of as an order to replenish inventory.

Depending on the destination of the fuel, the tow will travel through one or more set(s) of locks. The stochastic nature of the locking process is accounted for in the simulation model. Based on historical data, the locking times are assumed to follow a triangular distribution with a minimum of one hour, a maximum of three hours, and a mode of two hours.

Upon arriving at the lock, the tow attempts to seize a resource representing the lock. If this resource is busy, the tow waits in queue for passage through the lock. The locking time, or time required to traverse the lock, is a source of uncertainty in the model. The tow travels to its destination, seizing and delaying at locks as required. In this way, the tow is delayed for the appropriate travel time to and from its destination. When the tow arrives at its destination, each entity is then routed to the appropriate storage based on its attributes, where it decrements the total number of orders in the system and increments the level of inventory.

\section{AN OVERVIEW OF THE DECISION SUPPORT SYSTEM}

The decision support system developed for this application relies on the use of a heuristic optimization scheme based on scatter search, interfaced with the simulation model, for optimization of criterion model involving maximization of expected utility. The control variables are the reorder points and maximum capacities at each demand point in the system. The system also relies on the use of variance reduction techniques in order to improve the accuracy of the estimate of expected utility.

\subsection{The Criterion Model}

The criterion model is employed to guide the optimization process. The criterion model used for the optimization of the system described above is a single attribute utility function of the estimate of the expected value of the total solution cost (TSC) based on various costs associated with a particular vector of reorder point settings. The costs considered include the total penalty cost (TPC), variable transportation cost (VTC), and the total inventory holding cost (IHCTotal). The following paragraphs explain the calculations and implications of the aforementioned costs.

The total penalty cost (TPC) is the sum of the product of the number of backorders (BO) and the backorder cost (BOC) and the product of the penalty cost (PC) and the number of days with zero inventory (Penalty) as shown in below.

$$
\mathrm{TPC}=(\mathrm{BO} * \mathrm{BOC})+(\mathrm{PC} * \text { Penalty })
$$

After decrementing the inventory, the entity checks the level of the inventory versus the reorder point. If the inventory is below the reorder point, the entity then checks whether or not the inventory is negative. If the inventory is negative the entity increments the value of the variable $\mathrm{BO}$ by one. The values of $\mathrm{BOC}$ and $\mathrm{PC}$ are set and do not change during the simulation run.

The Variable Transportation Cost (VTC) is the product of a per mile transportation cost (PMTC) and the distance traveled (DT) as shown below.

$$
\mathrm{VTC}=\mathrm{PMTC} * \mathrm{DT}
$$

The value of PMTC is set and does not change during the simulation run.

The Inventory Holding Cost (IHCTotal) is the sum of the individual inventory holding costs for each of the six fuel types at each of the six locations. The individual IHC's are calculated as the product of three terms: the average level of inventory for a specific fuel at a specific location in units of barrels, a constant $h$ (a holding charge in 


\section{Anderson and Evans}

units of $\$ /$ barrel/day), and $D$ (the number of days simulated). The average level of inventory of fuel could be a negative number; therefore the individual holding cost for fuel type $i$ at location $j$ is calculated as shown below.

$$
\mathrm{IHC}_{i j}=\max \left(\mathrm{DAVG}_{\left.\left(\text {fuel }_{i} \text { at } \text { location }_{j}\right) * h * D, 0\right)}\right.
$$

IHCTotal is then simply the sum of the individual inventory holding costs over all fuel types and locations. Finally, the total solution cost (TSC) is calculated as the sum of TPC, VTC, and IHCTotal. The output from the simulation model, then is a single value describing the cost for a specific vector of reorder point settings.

\subsection{The Optimization Algorithm}

The optimization algorithm used for the DSS was scatter search, an evolutionary heuristic approach that has proven very effective for solving a diverse array of optimization problems. As noted by Glover, Kelly, and Laguna (1999), "Scatter search focuses on generating relevant outcomes without losing the ability to produce diverse solutions, due to the way the generation process is implemented." For example, newly generated points are not convex combinations of the initial points. These new points are extrapolations, containing information not contained in the initial reference points.

Scatter search is a metaheuristic that relies on the concepts of quality of a solution and diversity of solution sets. The quality of a solution refers to the value of the objective function, while the diversity of a solution set refers to the differences between pairs of solutions in the reference set. Requiring a specified level of diversity helps to assure that the procedure will not get trapped at a local optimum. The basic scatter search design can be described as follows.

The diversification generation method is employed to "generate a starting set of solutions to guarantee a critical level of diversity" (Laguna and Marti 2003). For the purpose of the diversification generation method, the application described herein divides the range of each decisionvariable into four subranges of equal size. A solution is then constructed in two steps; a subrange is randomly selected, and then a value is randomly generated from the selected subrange. The diversification generation method focuses on the diversity of the solutions and not the quality. Each solution is then passed to the improvement method.

The improvement method is used to improve the set of diverse solutions, thereby producing a set, denoted by $R$. The improvement method used for this application is Nelder and Mead's (1965) simplex method. This method is a classical numerical search technique for unconstrained nonlinear optimization problems. The improvement first constructs the current simplex for each diverse solution. The construction of the initial current simplex requires an initial point and a step factor, or distance between two vertices. The initial point is a point generated by the diversification generation method, while the step factor is input by the decision maker.

The improvement of diverse solutions is repeated until the set $R$ contains the number of unique members specified by the decision maker. The reference set is typically small, made up of no more than 20 solutions. The Reference Set Update Method is then invoked.

The Reference Set Update Method is used to designate a reference set of the best solutions. The best solutions are not based solely on objective function value. A solution may be added to the reference set if it improves the diversity of the set even when the objective function value of the solution is inferior. In this way the reference set includes both high quality and high diversity members from the improved solutions.

The decision maker specifies the size of the reference set, $b$, at the start of the procedure. The Reference Set Update Method forms two mutually exclusive subsets, $b_{I}$ and $b_{2}$ such that $b_{1} \bigcup b_{2}=b$, so $\left|b_{1}\right|+\left|b_{2}\right|=|b|$, where $b_{1}$ represents the subset of high quality solutions and $b_{2}$ represents the subset of high diversity solutions. The reference set, RefSet, is formed as follows: The set $R$ of improved diverse solutions is sorted in ascending order based on objective function value. The top $\left|b_{l}\right|$ solutions are added to RefSet and removed from $R$. The remaining $\left|b_{2}\right|$ members of RefSet are added based on a maximum value of minimum Euclidean distance as follows: For each remaining member of $R$, we calculate its Euclidean distance from each member of RefSet. The minimum Euclidean distance for each member of $R$ is stored. The member of the set $R$ with the maximum minimum Euclidean distance metric is removed from $R$ and added to RefSet. We then calculate the Euclidean distance for each remaining member of $R$ to each member of RefSet and repeat the process until the size of RefSet is that specified by the decision maker.

The Subset Generation Method consists of generating subsets from the reference set that will be used for creating new solutions. The subsets generated in the Subset Generation Method consist of all two element pairs. The number of this type of subset is $\left(|\mathrm{b}|^{2}-|b|\right) / 2$. These subsets are then combined using the Solution Combination Method. The Solution Combination Method is used to develop new solutions based on structured combinations of the subsets generated in the Subset Generation Method. These combinations are structured to create points "both inside and outside the convex regions spanned by the reference solutions" (Laguna and Marti 2003).

The individual subset solutions formed by the Solution Combination Method are then improved using the Improvement Method as previously described. 


\section{Anderson and Evans}

The reference set is then updated as previously described and the stopping criteria are checked. The improved solutions are sent to the Reference Set Update Method and the process iterates until the stopping criteria are met.

The stopping criteria can be based on the decision maker's preferences in terms of the membership of the reference set, the number of iterations, or the elapsed time. For example, when the reference set is updated, it is checked for new members. If all members or a specified number of members are new, the process is repeated. If no new members have been added, the process stops. The decision maker can alternatively specify the number of iterations after which the best solution found thus far will be selected. Finally, the decision maker can specify a time limit on the search. For the purposes of the applications described in this paper, the decision maker is asked to specify a number of iterations as the stopping criteria.

\subsection{Variance Reduction Techniques}

Each evaluation performed as part of the optimization procedure discussed above involves the employment of the simulation model. Several replications are made at each design point in order to obtain an estimate of the expected utility for that design point. In order to improve the accuracy of the estimates (i.e., reduce the variances), two common variance reduction techniques are employed.

The variance reduction techniques used in this research are common random numbers and antithetic variates. Common random numbers $(\mathrm{CRN})$ is perhaps the most widely used VRT. L'Ecuyer (1994) and (Janssens, Deceuninck, and Van Breedam 1995) explain that the CRN methodology is usually used to estimate the difference between the expected performance measures of multiple systems. The CRN method uses the same underlying uniform random numbers to drive the simulation and to make sure that these random numbers are used at exactly the same place for each system. The basic idea is that the random noise will be the same for both systems; therefore the observed differences between the systems will be due to their differences, not random noise. McGeoch (1992) further explains $\mathrm{CRN}$ as a technique that "reduces the variance in the difference of two output measures by making trials as similar as possible between the two measures."

The concept of antithetic variates (AV) resembles CRN. L'Ecuyer (1994) explains that the idea is that very unlucky events in the first simulation should be compensated by "antithetic" very lucky events in the second one and vice versa, thus reducing the variance on average. So, we run the model using a sequence of underlying IID uniform deviates, U's, to drive the simulation for computing the unbiased estimate of the mean, for example, then we drive the simulation using the antithetic sequence, (1 -
$U$ )'s, to compute another unbiased estimate of the mean. The average of these two then becomes our new estimate for the mean, which should have a smaller variance.

\section{ILLUSTRATIVE EXAMPLE}

In the example described in this section, the "Decision Maker" initializes the start of the operation associated with the decision support system by setting values for a series of parameters:

1. Initial inventory levels for all tanks.

2. Lower and upper bounds for tank inventory lev els.

3. Parameters for the Scatter Search and the Nelder and Mead Simplex Search schemes.

4. Parameter values associated with the barge-tow configurations, including the capacities of the tanks on the barges.

5. Type of utility function representing the prefer ence structure of the Decision Maker, basically defining whether the Decision Maker is risk averse, risk neutral, or risk seeking.

The simulation is set up for a one-year run in real time. For the risk averse case, a total of 142,238 replications at various design points, and expected utility was improved from an initial value of .915 to a final value of .973 through the search process.

\section{SUMMARY AND CONCLUSIONS}

The objective of this paper has been to show how criterion models can be incorporated into a simulation optimization methodology which also utilizes variance reduction techniques. As stated previously, criterion models can be implemented to take into account the decision maker's criteria and rank the outcomes of an experiment based on those criteria. We have included the programming and implementation of a heuristic search algorithm for optimization of these complex systems.

We have also explored the use of variance reduction techniques to enhance the efficiency of the optimization process. Specifically, the variance reduction techniques of antithetic variates and common random numbers were incorporated into the methodology.

Finally, we have included the development of a graphical user interface to aid the decision maker. By developing an interface, this research allows a decision maker to specify status information, parameterize a search methodology, and set a risk attitude. This interface also allows the decision maker to view the progress of the search numerically and graphically. 


\section{Anderson and Evans}

This paper has explored several previously undeveloped areas of research including the application of variance reduction techniques to the optimization of stochastic simulation models. We have also demonstrated the efficiency of optimization of criterion models to consider the trade-offs between multiple conflicting performance measures for a given system.

\section{ACKNOWLEDGEMENT}

This paper is based on the first author's dissertation (Anderson 2004).

\section{REFERENCES}

Anderson, N.P. 2004. Simulation optimization of logistics systems through the use of criterion models, Ph.D. Dissertation. 2004. Department of Industrial Engineering, University of Louisville, Louisville, Kentucky.

Glover, F., J.P. Kelly, and M. Laguna. 1999. New advances for wedding optimization and simulation. In Proceedings of the 1999 Winter Simulation Conference, The Association for Computing Machinery, 255-260, New York, NY.

Glover, F. and M. Laguna. 2000. Fundamentals of scatter search and path relinking. Con. and Cyb. 39 (3): 653684.

Janssens, G. K., W. Deceuninck, and A. Van Breedam. 1995. Opportunities of robust regression for variance reduction in discrete event simulation, Journal of Computational and Applied Mathematics, Vol. 64, No. 1-2: 163-176.

Kelton, W. D.,R.P. Sadowski, and D.T. Sturrock. 2004. Simulation With Arena, $3^{\text {rd }}$ Edition, McGrawHill, Boston, MA.

Laguna, M., and R. Marti. 2003. Scatter Search Methodology and Implementations in $C$. Kluwer Academic Publishers., Boston, MA.

L'Ecuyer, P. 1994. Efficiency improvement and variance reduction, 1994 Winter Simulation Conference Proceedings, The Association for Computing Machinery, 122-132, New York, NY.

McGeoch, C. 1992. Analyzing algorithms by simulation: variance reduction techniques and simulation speedups, ACM Computing Surveys, Vol. 24, No. 2: $195-$ 212.

Nelder, J.A. and R. Mead. 1965. A simplex method for function minimization. Computer Journal 7: 308-313.

\section{AUTHOR BIOGRAPHIES}

NICHOLAS P. ANDERSON is a process engineer for Mesoscale Diagnostics in Gaithersburg, Maryland. He has a B.S. degree in Mathematics from Loras College and an M.S. in Industrial Engineering and a Ph.D. in Industrial Engineering from the University of Louisville. He has worked as a consultant in Industrial Engineering and as a Tooling Engineer. His research interests include simulation modeling and analysis, multi-objective optimization, and decision analysis. He can be reached by e-mail at <npande31@gmail. com>.

GERALD W. EVANS is a Professor in the Department of Industrial Engineering at the University of Louisville. He has a B.S. degree in Mathematics, and M.S. and Ph.D. degrees in Industrial Engineering, all from Purdue University. Before entering academia, he worked as an Industrial Engineer for Rock Island Arsenal, and as a Senior Research Engineer for General Motors Research Laboratories. Besides simulation modeling and analysis, his research interests include multi-objective optimization, decision analysis, and discrete optimization. He can be reached by email at <gwevan01@louisville.edu>. 